\title{
LINEARLY UNBOUNDED TIMED PETRI NETS IN MODELLING AND PERFORMANCE EVALUATION
}

\author{
W.M. Zuberek \\ Department of Computer Science \\ Memorial University of Newfoundland \\ St. John's, Canada A1C 5S7
}

\begin{abstract}
A b s t r a c t
Linearly unbounded timed Petri nets are free-choice place/transition nets with exponentially distributed firing times associated with transitions of a net, in which the infinite state space is generated by a finite set of linear equations. This linear description can be used for reduction of the infinite state space into an equivalent finite representation that can easily be solved for stationary probabilities of states. Many performance measures can be obtained directly from the stationary solution.
\end{abstract}

\section{INTRODUCTION}

The three basic methods for evaluating the performance of a complex system are [Ferr]: (1) measuring the system during its operation, (2) generating the results by a simulation program that "imitates" the behaviour of the system, and (3) solving a mathematical model that captures the essential features of the system. The last of these methods is in many ways the most satisfying one since it may provide many insights into dependencies and relationships between system parameters and performance characteristics that are not easily produced by the other methods. However, mathematical modelling is also the most difficult approach as it quite often uses advanced mathematical theories to derive the exact or approximate results. Therefore a number of techniques have been proposed to simplify and "automate" mathematical modelling. Stochastic Petri nets and timed Petri nets are two examples of such techniques in which the basic Petri nets [Ager,Mura,Pete,Reis] are augmented (in two different ways) by "time" attributes in order to represent the behavior of the system accurately.

Petri nets are abstract models of systems with interacting, concurrent or parallel components. Multiprocessor systems, distributed databases, communication and computer networks are just a few examples of such systems. However, the original approach to Petri nets does not take into account the duration of modelled activities; the changes of "states" (or markings) of a net are instantaneous events which can represent the "causality" of events, but which neglect any temporal considerations. Consequently, such a model (called an "ordinary Petri net") is not complete enough for analysis and evaluation of systems performance. Several different concepts of "timed" Petri nets [HoVe,RaPh,Sif] and "stochastic" Petri nets [AMCB,DBCT, Moll] have been proposed by assigning (deterministic or stochastic) firing or enabling times to places or transitions of Petri nets.

Timed Petri nets considered in this paper are unbounded free-choice place/transition nets with stochastic firing times associated with transitions of a net. In timed Petri nets, the firing of an enabled transition is composed of three "conceptual" steps; the first (instantaneous) removes tokens from the input places, the second (temporal) "holds" the removed tokens within the transition for the duration of the firing time, and the third step (instantaneous) moves tokens to all transition's output places [Zub1]. The stochastic firing times mean that the firing time of each transition is a random variable with the (negative) exponential distribution. It can be shown that then the holding times of "states" [Zub1] (which describe distributions of tokens in places as well as firing transitions of a net) are also exponentially distributed random variables. The state transition system representing the behavior of a timed Petri net (or the state graph of a net) is thus a discrete-state continuous-time Markov process which, for an unbounded net, has infinitely many states. Therefore, in order to effectively find the stationary properties of states, this infinite state space must be somehow reduced to an equivalent finite representation. For linearly unbounded nets, the infinite state space is generated by a finite number of linear equations each of which represents a class of "similar" states. The classes of "similar" states are infinite, however, the stationary probabilities are geometrically distributed in such classes, so each class can easily be aggregated into a single "superstate". Since the number of classes is finite, known methods can be used to find the stationary probabilities for "aggregated" states, and this also determines the stationary probability distributions within the classes.

Many probability measures can easily be derived from stationary probabilities of the states.

The main part of the paper contains three sections. Section 2 recalls the most important concepts and definitions for ordinary and timed Petri nets. Some properties of unbounded nets are presented in section 3 , and illustrated in section 4 with a simple model of an open queueing network. A few conclusions are given in section 5 .

\section{TIMED NETS}

An (ordinary) place/transition net $\mathbf{N}$ is a triple $\mathbf{N}=$ $(P, T, A)$ where $P$ is a (finite, nonempty) set of places, $T$ is a (finite, nonempty) set of transitions, and $A$ is a set of directed arcs, $A \subset P \times T \cup P \times T$. For each element of $P$ (and $T$ ), the input and output sets denote all those elements of $T$ (and $P$ ) which are connected by directed arcs to and from this element, respectively, i.e., $\operatorname{In} p(p)=\{t \in T \mid(t, p) \in A\}$, etc.

A place $p$ is shared if its output set contains more than one transition. A shared place $p$ is free-choice (or extended freechoice) iff all transitions sharing it have identical input sets, i.e., iff:

$$
\forall\left(t_{i}, t_{j} \in O u t(p)\right) \operatorname{Inpt}\left(t_{i}\right)=\operatorname{Inp}\left(t_{j}\right)
$$

A net is free-choice iff all its shared places are free-choice. Only free-choice net are considered in this paper.

A marked net $\mathbf{M}$ is a pair $\mathbf{M}=\left(\mathbf{N}, m_{0}\right)$, where $\mathbf{N}$ is a place/ transition net and $m_{0}$ is the initial marking function which assigns a nonnegative number of so called "tokens" to each place of a net, $m_{0}: P \rightarrow\{0,1,2, \ldots\}$. In a marked net a transitions $t$ is enabled if all its input places contain at least one token.

Each enabled transition can fire. When a transition $t$ fires, a token is removed from each of its input places (simultaneously), and a single token is added to each $t$ 's output place. This determines a new marking function of a net, a new set of enabled transitions, etc. The set of all marking functions that can be generated by a net is denoted by $M(\mathbf{M})$. 
A place $p$ of a marked net $\mathbf{M}$ is bounded iff there exists a bound on the number of tokens that any marking function of the set $M(\mathbf{M})$ can assign to $p$ :

$$
\exists(k>0) \forall(m \in M(\mathbf{M})) m(p)<k
$$

A marked net is bounded iff all its places are bounded. The set $M(\mathbf{M})$ of a bounded net is finite.

In marked nets, the firings of transitions are instantaneous events, and analysis of such nets usually assumes that the firings are performed "one at a time". Modeling of "real" systems must also take into account the duration of systems activities represented by transition firings. Therefore in timed nets [Zub1] each transition takes a "real time" to fire, and at any instant of time the tokens are distributed in places as well as (firing) transitions of a net. The "state" description is similar to marking functions of marked nets, but it contains the second component that describes the firing transitions. Moreover, to provide unambiguous modelling capability, in timed nets each firing starts at the same instant of time at which the transition becomes enabled (but some enabled transitions are disabled without firing, e.g., conflicting transitions in freechoice classes).

A timed net $\mathbf{T}$ is a triple, $\mathbf{T}=(\mathbf{M}, c, f)$, where $\mathbf{M}$ is a marked place/transition net, $c$ is a choice function, $c: T \rightarrow$ $[0,1]$, such that for each free-choice place $p, \sum_{t \in O u t(p)} c(t)=1$, and for all transitions $t$ that do not belong to free-choice classes, $c(t)=1$. It is assumed that the choices within freechoice classes of enabled transitions are independent random variables with discrete distributions described by corresponding probabilities $c(t)$.

A state of a timed net $\mathbf{T}$ is a pair of functions $s=(m, n)$, $m: P \rightarrow\{0,1, \ldots\}, n: T \rightarrow\{0,1, \ldots\}$, which describe the distribution of tokens in places $(m)$ and firing transitions $(n)$. The derivation of the state space of a timed net is described in [Zub2]; the state space of net $\mathbf{T}$ is denoted by $S(\mathbf{T})$.

A timed net $\mathbf{T}$ is bounded iff

$\exists(k>0) \forall(s=(m, n) \in S(\mathbf{T}))$

$\forall(p \in P) m(p)<k \wedge \forall(t \in T) n(t)<k$.

$\mathbf{T}$ is place-unbounded if there is no bound on the marking component $m$ of states $s=(m, n) \in S(\mathbf{T})$, and it is transitionunbounded if there is no bound on the firing component $n$ of states.

It can easily be shown that any firing sequence of a timed net $\mathbf{T}=(\mathbf{M}, c, f)$ is also a firing sequence in the marked net $\mathbf{M}$ (but the opposite is not true [Zub1]); this is due to the fact that in timed nets some tokens are associated with firing transitions and are "inactive" during the firing periods. Consequently, a bounded timed net can have unbounded marked net, but an unbounded timed net can never have a bounded marked net.

\section{UNBOUNDED NETS}

Each place/transition net $\mathbf{N}=(P, T, A)$ can be represented by an incidence (or connectivity) matrix $\mathbf{C}: P \times T \rightarrow$ $\{-1,0,+1\}$ in which places correspond to rows, transitions to columns, and the entries are defined as:

$\forall(p \in P) \forall(t \in T) \mathbf{C}[p, t]= \begin{cases}-1, & \text { if }(p, t) \in A \wedge(t, p) \notin A, \\ +1, & \text { if }(t, p) \in A \wedge(p, t) \notin A, \\ 0, & \text { otherwise. }\end{cases}$

It can be verified that if a marking function $m_{j}$ is obtained from another marking function $m_{i}$ by firing a transition $t_{k}$ then (in vector notation) $m_{j}=m_{i}+\mathbf{C}[., k]$, where $\mathbf{C}[., k]$ denotes the $k$-th column of $\mathbf{C}$, i.e., the column corresponding to $t_{k}$.

Incidence matrices disregard "selfloops", that is pairs of arcs $(p, t)$ and $(t, p)$; for such a selfloop any firing of a transition $t$ cannot change the marking of $p$; selfloops are neutral with respect to token count of a net. A pure net is defined as a net without selfloops [Reis].
Furthermore, it can be observed that in an ordinary (marked) net $\mathbf{M}$, if a marking $m_{j}$ is reachable from another marking $m_{i}$ by a firing sequence $\sigma=\left(t_{i_{1}} t_{i_{2}} \ldots t_{i_{k}}\right)$, and if $m_{j}$ is (componentwise) greater or equal to $m_{i}, m_{j} \geq m_{i}$, then:

- $m_{j}=m_{i}+\mathbf{C} \times \mathbf{I}_{\sigma}$, where $\mathbf{I}_{\sigma}$ is a $n_{t}$-element vector $\left(n_{t}\right.$ is the number of transintions in the set $\mathbf{T}$ ) whose $j$-th element $\mathbf{I}_{\sigma}[j]$ is equal to the number of occurrences of transition $t_{j}$ in the sequence $\sigma$.

- $\sigma$ is a feasible firing sequence in $m_{j}$, i.e., $t_{i_{1}}$ is enabled by $m_{j}$ so it can fire, $t_{i_{2}}$ is enabled by the marking created by firing $t_{i_{1}}$ in $m_{j}$, etc.

- Consequently, there exists yet another marking $m_{k}$ that is reachable from $m_{j}$ by $\sigma$, so $m_{k}=m_{j}+\mathbf{C} \times \mathbf{I}_{\sigma}$.

- Applying the same reasoning to $m_{k}$ and $m_{j}$ rather than $m_{j}$ and $m_{i}$, it follows that the marking $m_{k}+\mathbf{C} \times \mathbf{I}_{\sigma}$ is feasible in $m_{k}$, as well as $m_{k}+2 * \mathbf{C} \times \mathbf{I}_{\sigma}, m_{k}+3 * \mathbf{C} \times \mathbf{I}_{\sigma}$, etc.

In conclusion, in a marked net $\mathbf{M}$, if a marking $m_{j}$ is reachable from another marking $m_{i}$, and $m_{j}$ is (componentwise) greater or equal to $m_{i}$, and $m_{j} \neq m_{i}$, then $\mathbf{M}$ is unbounded. For marked nets this property is a sufficient as well as necessary condition of unboundedness [Reis].

In timed nets the firing of a transition is not an "instantaneous" event, and therefore a different description of net structure is needed, in which the beginnings and the terminations of firings are separated as this new description must correspond to state transitions rather than transformations of marking functions.

A place/transition net $\mathbf{N}$ is represented by a pair of $n_{p} \times n_{t}$ matrices, the input incidence matrix $\mathbf{C}^{-}: P \times T \rightarrow\{-1,0\}$ which is defined as:

$$
\forall(p \in P) \forall(t \in T) \mathbf{C}^{-}[p, t]= \begin{cases}-1, & \text { if }(p, t) \in A, \\ 0, & \text { otherwise. }\end{cases}
$$

and the output incidence matrix $\mathbf{C}^{+}: P \times T \rightarrow\{0,+1\}$ defined as:

$$
\forall(p \in P) \forall(t \in T) \mathbf{C}^{+}[p, t]= \begin{cases}+1, & \text { if }(t, p) \in A \\ 0, & \text { otherwise. }\end{cases}
$$

It can be verified that if a state $\left(m_{j}, n_{j}\right)$ is reached from another state $\left(m_{i}, n_{i}\right.$ by termination of a firing of transition $t_{k}$ and then initiating firings of transitions $t_{i_{l}}, l=1, \ldots, n$, than

$$
\begin{array}{r}
m_{j}=m_{i}+\mathbf{C}^{+}[., k]+\sum_{1 \leq l \leq n} \mathbf{C}^{-} \\
n_{j}=n_{i}-\mathbf{1}_{\mathbf{k}}+\sum_{1 \leq l \leq n} \mathbf{1}_{\mathbf{i}_{\mathbf{l}}},
\end{array}
$$

where $\mathbf{1}_{\mathbf{k}}$ denotes a vector with only one nonzero $k$-th element that is equal to 1 .

Furthermore, if a state $s_{j}=\left(m_{j}, n_{j}\right)$ can be (generally) reached from another state $s_{i}=\left(m_{i}, n_{i}\right)$ through a sequence $\sigma$ of intermediate states $\sigma=\left(s_{i_{1}}, \ldots, s_{i_{n}}\right)$ such that the total numbers of transitions that terminate their firings in this sequence are described by a vector $\mathbf{I}^{+}$while another vector $\mathbf{I}^{-}$ describes the numbers of transitions that initiated their firings in $\sigma$ (the two vectors may be different), than:

$$
\begin{gathered}
m_{j}=m_{i}+\mathbf{C}^{+} \times \mathbf{I}^{+}+\mathbf{C}^{-} \times \mathbf{I}^{-}, \\
n_{j}=n_{i}-\mathbf{I}^{+}+\mathbf{I}^{-} .
\end{gathered}
$$

A timed net is place-unbounded if there exist two nonnegative vectors, an $n_{t}$-element vector $\mathbf{I}$ and an $n_{p}$-element vector J such that: 
1. $\left(\mathbf{C}^{+}+\mathbf{C}^{-}\right) \times \mathbf{I}=\mathbf{J}$,

2. the vectors $\mathbf{I}$ and $\mathbf{J}$ are $t$-feasible, that is, there exist a state $s_{i}=\left(m_{i}, n_{i}\right)$ and a state $s_{j}=\left(m_{i}+\mathbf{J}, n_{i}\right)$ reachable from $s_{i}$ by a sequence of state transitions in which the total numbers of transition firings are described by $\mathbf{I}$. The vector $\mathbf{J}$ is called the marking increment vector.

A timed net is transition-unbounded if there exist two nonnegative $n_{t}$-element vectors $\mathbf{I}$ and $\mathbf{J}$ such that:

1. $\mathbf{C}^{-} \times \mathbf{I}+\mathbf{C}^{+} \times(\mathbf{I}+\mathbf{J})=\mathbf{0}$,

2 . the vectors $\mathbf{I}$ and $\mathbf{J}$ are $p$-feasible, that is, there exist a state $s_{i}=\left(m_{i}, n_{i}\right)$ and a state $s_{j}=\left(m_{i}, n_{i}+\mathbf{J}\right)$ reachable from $s_{i}$ by a sequence of state transitions in which the total numbers of initiated transition firings are described by $\mathbf{I}$, and the total numbers of terminated firings correspond to $\mathbf{I}-\mathbf{J}$. The vector $\mathbf{J}$ is called the firing increment vector.

A timed net $\mathbf{T}$ is linearly unbounded if it is place-unbounded and its all place increment vectors $\mathbf{J}$ contain only one non-zero element.

The following section illustrates these concepts in greater detail.

\section{PERFORMANCE EVALUATION}

Performance evaluation of unbounded nets is illustrated by a simple net model shown in Fig.1. It is an open network model of a system in which a "source" with exponentially distributed interarrival times is represented by $p_{1}$ and $t_{1}$ (the arrival rate $r\left(t_{1}\right)$ is equal to 1 arrival per time unit), and the remaining part of the net models a (single channel) server composed of two consecutive stages. The first stage $\left(t_{2}\right.$ and $\left.t_{3}\right)$ provides service with a hyperexponential distribution; the service rate is equal to 2 with probability $0.25\left(t_{2}\right)$ and 5 with probability $0.75\left(t_{3}\right)$; the place $p_{2}$ is a free-choice place. Service times of the second stage $\left(t_{4}\right)$ are exponentially distributed with the rate equal to 4. The total service time is thus hypoexponentially distributed with corresponding parameters.

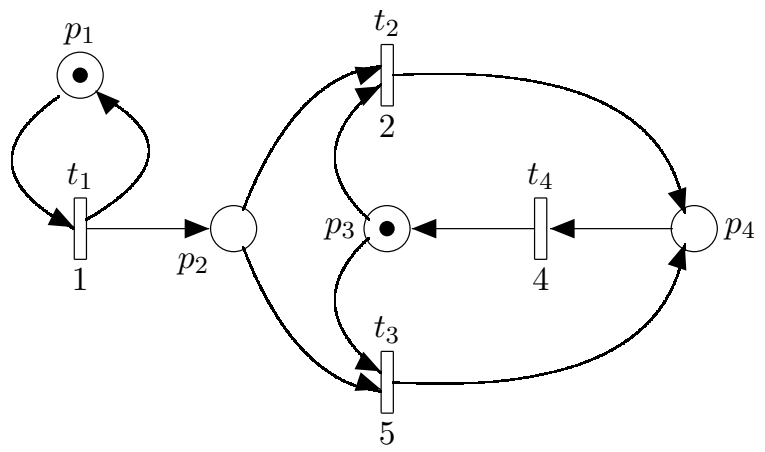

Fig.1. Unbounded M-timed Petri net T.

The incidence matrices $\mathbf{C}, \mathbf{C}^{-}$and $\mathbf{C}^{+}$are as follows:

\begin{tabular}{c|cccc}
$\mathbf{C}$ & $t_{1}$ & $t_{2}$ & $t_{3}$ & $t_{4}$ \\
\hline$p_{1}$ & 0 & 0 & 0 & 0 \\
$p_{2}$ & +1 & -1 & -1 & 0 \\
$p_{3}$ & 0 & -1 & -1 & +1 \\
$p_{4}$ & 0 & +1 & +1 & -1
\end{tabular}

\begin{tabular}{|c|c|c|c|c|c|c|c|c|c|}
\hline $\mathbf{C}^{-}$ & $t_{1}$ & $t_{2}$ & $t_{3}$ & $t_{4}$ & $\mathbf{C}^{+}$ & $t_{1}$ & $t_{2}$ & $t_{3}$ & $t_{4}$ \\
\hline$p_{1}$ & -1 & 0 & 0 & 0 & $p_{1}$ & +1 & 0 & 0 & 0 \\
\hline$p_{2}$ & 0 & -1 & -1 & 0 & $p_{2}$ & +1 & 0 & 0 & 0 \\
\hline$p_{3}$ & 0 & -1 & -1 & 0 & $p_{3}$ & 0 & 0 & 0 & +1 \\
\hline$p_{4}$ & 0 & 0 & 0 & -1 & $p_{4}$ & 0 & +1 & +1 & 0 \\
\hline
\end{tabular}

It can be verified that for $\mathbf{I}=[1,0,0,0], \mathbf{C} \times \mathbf{I}=[0,1,0,0]$, which means that each firing of $t_{1}$ increases the marking of $p_{2}$ by one token; $p_{2}$ is an unbounded place and, consequently, the marked net shown in Fig.1 also is unbounded.

The timed net is also unbounded, which can be checked by verifying the feasibility condition. For example, in the state $s_{2}$ from Tab.1, $\left(m_{2}, n_{2}\right)=([0,0,0,0],[1,0,1,0])$, the termination of the firing of $t_{1}$ deposits single tokens in $p_{1}$ and $p_{2}$, and since $p_{1}$ is the only input place of $t_{1}, t_{1}$ immediately starts its firing again (there are no other enabled transitions). So, for the firing described by $\mathbf{I}$, the marking increment vector is equal to $\mathbf{J}$. The timed net is place unbounded, and it is linearly unbounded.

Because of the unbounded place $p_{2}$, the state space of this net is infinite, but the states which are "distant" from the initial state are not very likely to be active. Since the firing times of both $t_{2}$ and $t_{3}$ are exponentially distributed random variables, the probability that there will be $k$ firings of $t_{1}$ before a termination of $t_{2}$ or $t_{3}$ firing is nonzero, and this is true for any value of $k$. Therefore, the state space of $\mathbf{T}$ will contain a series of states differing only in the marking of $p_{2}$.

The initial part of the state graph of $\mathbf{T}$ is shown in Fig.2 (the numbering of states is quite irrelevant; actually it is generated by a net analyzing program, which uses a rather complicated scheme for assigning consecutive state numbers.)

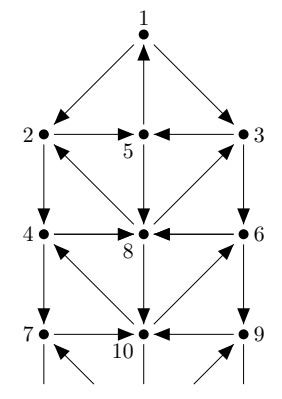

Fig.2. State graph of $\mathbf{T}$.

The state graph has a regular structure which reflects the unboundedness condition. The state $s_{2}$, using the feasibility condition, derives the states $s_{4}=\left(m_{2}+\mathbf{J}, n_{2}\right), s_{7}=$ $\left(m_{4}+\mathbf{J}, n_{2}\right)$, etc. Similarly, $s_{3}$ derives $s_{6}=\left(m_{3}+\mathbf{J}, n_{3}\right)$, $s_{9}=\left(m_{6}+\mathbf{J}, n_{3}\right)$, etc., and $s_{5}$ derives $s_{8}=\left(m_{5}+\mathbf{J}, n_{5}\right)$, $s_{1} 0=\left(m_{8}+\mathbf{J}, n_{5}\right)$, etc. The state space can thus be represented by a linear form $S\left(\mathbf{T}=\left\{s_{\}} \cup\left\{\left(m_{i}+k * \mathbf{J}, n_{i}\right), i=\right.\right.\right.$ $2,3,4 \wedge k=0,1,2, \ldots\}$. Since the "replicated" states are interrelated in the same way as are the "basic" states, state graph of $\mathbf{T}$ contains a regular (three-state) section $\left\{s_{4}, s_{8}, s_{6}\right\}$, $\left.\left\{s_{7}, s_{10}, s_{9}\right\}, \ldots\right)$ that is repeatedly added to a four-state "basis" $\left\{s_{1}, s_{2}, s_{5}, s_{3}\right\}$. These added sections form "layers" of the state graph which can be "folded" or reduced to a single layer with stationary probabilities that are sums of corresponding infinite geometric series.

The derivation of the (reduced) state space for $\mathbf{T}$ is shown in Tab.1, in which $s_{i}$ and $s_{j}$ are the present and the next states, respectively, $m_{i}$ and $n_{i}$ describe the distributions of tokens in places $\left(m_{i}\right)$ and firing transitions $\left(n_{i}\right), h\left(s_{i}\right)$ if the average holding time in the state $s_{i}$, and $q\left(s_{i}, s_{j}\right)$ is the probability of transition from the state $s_{i}$ to the state $s_{j}$.

The states in Tab.1 are "automatically" subdivided into groups. The first group contains all initial states. Whenever linear unboundedness is detected (by checking "regularity" of the created state space), the second group of states is formed which contains repetitions of some states from the first group; in Tab. 1 this second group is indicated by the star symbols (the states "*7(4)", "* $9(6)$ " and "* $10(8)$ ") with the corresponding "first" states given in parentheses. Similarly, the states "***11", "** 12 " and "** 13 " form the third group, derived from the second group, etc. 


\begin{tabular}{|c|c|c|c|c|c|c|}
\hline$s_{i}$ & $\begin{array}{c}m_{i} \\
1234 \\
\end{array}$ & $\begin{array}{c}n_{i} \\
1234 \\
\end{array}$ & $h\left(s_{i}\right)$ & $t_{k}$ & $s_{j}$ & $q\left(s_{i}, s_{j}\right)$ \\
\hline \multirow[t]{2}{*}{1} & $\begin{array}{lllll} & 0 & 1 & 0\end{array}$ & 10000 & 1.000 & 1 & 2 & 0.750 \\
\hline & & & & & 3 & 0.250 \\
\hline \multirow[t]{2}{*}{2} & $\begin{array}{llll}0 & 0 & 0 & 0\end{array}$ & 1010 & 0.167 & 1 & 4 & 0.167 \\
\hline & & & & 3 & 5 & 0.833 \\
\hline \multirow[t]{2}{*}{3} & $\begin{array}{llll}0 & 0 & 0 & 0\end{array}$ & 1100 & 0.333 & 1 & 6 & 0.333 \\
\hline & & & & 2 & 5 & 0.667 \\
\hline \multirow[t]{2}{*}{4} & 0100 & 1010 & 0.167 & 1 & $* 7$ & 0.167 \\
\hline & & & & 3 & 8 & 0.833 \\
\hline \multirow[t]{2}{*}{5} & $\begin{array}{llll}0 & 0 & 0 & 0\end{array}$ & 1001 & 0.200 & 1 & 8 & 0.200 \\
\hline & & & & 4 & 1 & 0.800 \\
\hline \multirow[t]{2}{*}{6} & 0100 & 1100 & 0.333 & 1 & $* 9$ & 0.333 \\
\hline & & & & 2 & 8 & 0.667 \\
\hline \multirow[t]{3}{*}{8} & 0100 & 1001 & 0.200 & 1 & $* 10$ & 0.200 \\
\hline & & & & 4 & 2 & 0.600 \\
\hline & & & & & 3 & 0.200 \\
\hline \multirow[t]{2}{*}{$7(4)$} & 0200 & 1010 & 0.167 & 1 & $* * 11$ & 0.167 \\
\hline & & & & 3 & $* 10$ & 0.833 \\
\hline \multirow[t]{2}{*}{$9(6)$} & 0200 & 1100 & 0.333 & 1 & $* * 12$ & 0.333 \\
\hline & & & & 2 & $* 10$ & 0.667 \\
\hline \multirow[t]{3}{*}{$10(8)$} & 0200 & 1001 & 0.200 & 1 & $* * 13$ & 0.200 \\
\hline & & & & 4 & 4 & 0.600 \\
\hline & & & & & 6 & 0.200 \\
\hline
\end{tabular}

Tab.1. The set of reachable states for $\mathbf{T}$.

The infinite set of states $S(\mathbf{T})$ can thus be subdivided into four disjoint classes of states (Fig.2 and Tab.1), $S_{0}$ that contains all "basic" states of $\mathbf{T}$ (i.e., $s_{1}, s_{2}, s_{3}$ and $s_{5}$ ), $S_{1}$ that represents the "bottom" layer of folding $\left(s_{4}, s_{6}\right.$ and $\left.s_{8}\right), S_{2}$ that represents the "second" layer of folding $\left(s_{7}, s_{9}\right.$ and $\left.s_{10}\right)$, and all remaining states. Since the stationary probabilities of folded states in consecutive layers are geometrically distributed, the infinite sum of probabilities can be replaced be the sums of corresponding geometrical series with the quotient of these series (denoted by $\rho$ ) as an additional "new" unknown. This leads to a system of simultaneous nonlinear (in fact, quadratic for single-place unbounded place nets) equilibrium equations in which $x(s)$ denotes the (unknown) stationary probability of the state $s$ :

$$
\left\{\begin{array}{l}
\sum_{s_{j} \in S_{0} \cup S_{1}} h\left(s_{j}\right) * q\left(s_{j}, s_{i}\right) * x\left(s_{j}\right)=h\left(s_{i}\right) * x\left(s_{i}\right) ; s_{i} \in S_{0} \\
\sum_{s_{j} \in S_{0} \cup S_{1}} h\left(s_{j}\right) * q\left(s_{j}, s_{i}\right) * x\left(s_{j}\right)+ \\
{[0.5 i n] \sum_{s_{j} \in S_{2}} h\left(s_{j}\right) * q\left(s_{j}, s_{i}\right) * \rho * x\left(s_{j}\right)=h\left(s_{i}\right) * x\left(s_{i}\right) ; s_{i} \in S_{1}} \\
(1-\rho) \sum_{s_{i} \in S_{0}} x\left(s_{i}\right)+\sum_{s_{j} \in S_{1}} x\left(s_{j}\right)=1-\rho
\end{array}\right.
$$

For $\mathbf{T}$ this system contains 8 equations ( 4 for $S_{0}, 3$ for $S_{1}$ and the "normalizing" equation), and the solution is $\rho=0.483, x\left(s_{1}\right)=0.472, \quad x\left(s_{2}\right)=0.093, \quad x\left(s_{3}\right)=0.062$, $x\left(s_{4}\right)=0.032, x\left(s_{5}\right)=0.118, x\left(s_{6}\right)=0.032$ and $x\left(s_{8}\right)=0.068$. The remaining probabilities can be obtained from recursive formulas, e.g., $x\left(s_{7}\right)=\rho * x\left(s_{4}\right)$, etc.

Many performance measures can be derived from stationary probabilities of the states. For example, the utilization of the server corresponds to the probability $1-\operatorname{Prob}($ serveridle); the server is idle in all those states $s_{i}$ in which $m_{i}\left(p_{3}\right)=1$. For the net from Fig.1, the only state with idle server is $s_{1}$ (see Tab.1), the utilization of the server is equal to $1-x\left(s_{1}\right)=0.528$. The probability that there are at least two jobs waiting for processing is the sum of stationary probabilities of all those states in which $m_{2} \geq 2$, which is equal to 0.123 for this model, and so on.

\section{CONCLUDING REMARKS}

A systematic approach to analysis of a class of unbounded timed Petri nets has been presented. The characteristic property of this class of nets is that their state spaces can be generated by a finite set of linear "state" equations. This approach opens a new direction in applications of timed Petri net models as all existing approaches [AMCB,DBCT,HoVe,RaPh] assume that the modelling nets are bounded, and their state spaces are finite.

The approach can be used efficiently during generation of the state space of a timed net; whenever a new state is created, conditions for unboundedness can be checked, an the infinite classes of states reduced, as shown in Tab.1.

The presented approach may seem rather complicated and thus impractical, it should be noted, however, that it can easily be implemented as a computer program, and then all the detailed state descriptions and state transitions can be "invisible" for users.

\section{Acknowledgement}

The Natural Sciences and Engineering Research Council of Canada partially supported this research through Operating Grant A8222.

\section{$R$ e f e r e n c e s}

[Ager] T. Agerwala, "Putting Petri nets to work"; IEEE Computer Magazine, vol.12, no.12, pp.85-94, 1979.

[AMCB] M. Ajmone Marsan, G. Conte, G. Balbo, "A class of generalized stochastic Petri nets for the performance evaluation of multiprocessor systems"; ACM Trans. on Computer Systems, vol.2, no.2, pp.93-122, 1984.

[DBCT] J.B. Dugan, A. Bobbio, G. Ciardo, K. Trivedi, "The design of a unified package for the solution of stochastic Petri net models"; Proc. Int. Workshop on Timed Petri Nets, Torino, Italy, pp.6-13, 1985.

[Ferr] D. Ferrari, "Computer systems performance evaluation"; Prentice-Hall 1978.

[HoVe] M.A. Holliday, M.K. Vernon, "A generalized timed Petri net model for performance evaluation"; Proc. Int. Workshop on Timed Petri Nets, Torino, Italy, pp.181-190, 1985.

[Moll] M.K. Molloy, "Performance analysis using stochastic Petri nets"; IEEE Trans. on Computers, vol.31, no.9, pp.913-917, 1982.

[Mura] T. Murata, "Petri nets: properties, analysis and applications"; Proceedings of the IEEE, vol.77, no.4, pp.541580,1989

[Pete] J.L. Peterson, "Petri net theory and the modeling of systems", Prentice-Hall 1981.

[RaPh] R.R. Razouk, C.V. Phelphs, "Performance analysis using timed Petri nets"; in: "Protocol Specification, Testing, and Verification IV" (Proc. of the IFIP WG 6.1 Fourth Int. Workshop, Skytop Lodge PA, June 11-14, 1984); Y. Yemini, R. Strom, S. Yemini (eds.), pp.561-576, North-Holland 1985.

[Sif] J. Sifakis, "Use of Petri nets for performance evaluation"; in: "Measuring, modelling and evaluating computer systems", pp.75-93, North-Holland 1977.

[Zub1] W.M. Zuberek, "M-timed Petri nets, priorities, preemptions, and performance evaluation of systems"; in: "Advances in Petri Nets 1985" (Lecture Notes in Computer Science 222), G. Rozenberg (ed.), pp.478-498, Springer Verlag 1986.

[Zub2] W.M. Zuberek, QOn generation of state space for timed Petri netsU; Proc. ACM Annual Computer Science Conf., Atlanta GA, pp.239-248, 1988. 